arXiv:09. . [hep-th]

\title{
Bending AdS Waves with New Massive Gravity
}

\author{
Eloy Ayón-Beato, 1, 因 Gaston Giribet, 2,3 , 田 and Mokhtar Hassaïne, \\ ${ }^{1}$ Departamento de Fúsica, CINVESTAV-IPN, Apdo. Postal 14-740, 07000, México D.F., México. \\ ${ }^{2}$ Departamento de Física, Universidad de Buenos Aires FCEN - UBA and CONICET, Argentina. \\ ${ }^{3}$ Abdus Salam International Centre for Theoretical Physics ICTP, Trieste, Italy. \\ ${ }^{4}$ Instituto de Matemática y Física, Universidad de Talca, Casilla 747, Talca, Chile.
}

\begin{abstract}
We study AdS-waves in the three-dimensional new theory of massive gravity recently proposed by Bergshoeff, Hohm, and Townsend. The general configuration of this type is derived and shown to exhibit different branches, with different asymptotic behaviors. In particular, for the special fine tuning $m^{2}= \pm 1 /\left(2 l^{2}\right)$, solutions with logarithmic fall-off arise, while in the range $m^{2}>-1 /\left(2 l^{2}\right)$, spacetimes with Schrödinger isometry group are admitted as solutions. Spacetimes that are asymptotically $\mathrm{AdS}_{3}$, both for the Brown-Henneaux and for the weakened boundary conditions, are also identified. The metric function that characterizes the profile of the AdS-wave behaves as a massive excitation on the spacetime, with an effective mass given by $m_{\mathrm{eff}}^{2}=m^{2}-1 /\left(2 l^{2}\right)$. For the critical value $m^{2}=-1 /\left(2 l^{2}\right)$, the value of the effective mass precisely saturates the Breitenlohner-Freedman bound for the $\mathrm{AdS}_{3}$ space where the wave is propagating on. The analogies with the AdS-wave solutions of topologically massive gravity are also discussed. Besides, we consider the coupling of both massive deformations to Einstein gravity and find the exact configurations for the complete theory, discussing all the different branches exhaustively. One of the effects of introducing the Chern-Simons gravitational term is that of breaking the degeneracy in the effective mass of the generic modes of pure New Massive Gravity, producing a fine structure due to parity violation. Another effect is that the zoo of exact logarithmic specimens becomes considerably enlarged.
\end{abstract}

PACS numbers:

\section{INTRODUCTION}

A new theory of massive gravity in three dimensions has been recently proposed by Bergshoeff, Hohm, and Townsend [1]. At the linearized level, this theory has been shown to be equivalent to the three-dimensional Fierz-Pauli action for a massive spin-2 field, which turns out to be unitary. In contrast with the Topologically Massive Gravity [2, 3], this new theory of gravity is parity invariant. Following the authors of [4, [5, 6], we will refer to this theory as the New Massive Gravity. As it is the case for Topologically Massive Gravity, the New Massive Gravity entails higher order modifications to the three-dimensional General Relativity with the consequence that the graviton excitations of both theories become massive through similar mechanisms.

This New theory of Massive Gravity has attracted much attention recently. In Refs. 7, 8] the unitarity of the model was discussed; in Ref. [4] exact solutions representing asymptotically warped- $\mathrm{AdS}_{3}$ black holes were found; other interesting solutions are analyzed in [9]. In Refs. [5, 6], the boundary conditions for the theory in asymptotically $\mathrm{AdS}_{3}$ spaces were studied. In particular, it was argued in Ref. [6] that, for a particular relation between the cosmological constant and the mass parameter of the theory, the concept of asymptotically $\mathrm{AdS}_{3}$ boundary conditions is compatible with a weakened logarithmic

\footnotetext{
${ }^{*}$ Electronic address: ayon-beato-at-fis.cinvestav.mx

${ }^{\dagger}$ Electronic address: gaston-at-df.uba.ar

${ }^{\ddagger}$ Electronic address: hassaine-at-inst-mat.utalca.cl
}

fall-off at large distance. This amounts to relax the standard Brown-Henneaux asymptotic conditions for gravity in $\mathrm{AdS}_{3}$ space [10], and it is analogous to what happens in Topologically Massive Gravity at the chiral point [11, 12]. In the case of Topologically Massive Gravity, the feasibility of considering weakened $\mathrm{AdS}_{3}$ boundary conditions allows to reinterpret some of the AdS-waves with logarithmic profile found in Refs. [13, 14] as being asymptotically $\mathrm{AdS}_{3}$ spacetimes. These configurations, together with those that do not exhibit the logarithmic decay, ${ }^{1}$ have been reconsidered recently [17, 18] within the context of the revived interest in Topologically Massive Gravity [19, 20, 21, 22, 23].

Here, we will explore the AdS-wave configurations of the New Massive Gravity. In particular, this will allow us to study the dynamics of the theory beyond the linearized level and to discuss the different asymptotic behaviors; a strategy proved to be useful in Topologically Massive Gravity [14]. For example, we will find that solutions with logarithmic fall-off arise for certain values of the parameters. The existence of such solutions to New Massive Gravity is suggested by the linearized analysis performed in Ref. [6]. Nevertheless, in this paper we go beyond the perturbative analysis and find exact solutions of this kind. More generally, we will exhibit a

\footnotetext{
${ }^{1}$ See Ref. [15] for a preliminary derivation of the no-logarithmic branch, where it was argued that in order to be supersymmetric the solutions should not depend on the retarded time. See also Ref. [16], where the interpretation as AdS-waves was first given to the final forms of the metric originally derived in Ref. [13].
} 
whole family of AdS-wave solutions of the New Massive Gravity; and these solutions are, in some sense, the analogues of those arising in Topologically Massive Gravity $[13,14,15,16,17,18]$. For a particular range of the parameters, we will show that the isometry of the solutions coincides with the Schrödinger symmetry. We will also analyze similar considerations for a more general theory given by the coupling between both massive gravity theories.

The paper is organized as follows. In Sec. III we provide a brief introduction to AdS-wave configurations. Section [II is devoted to present the New Massive Gravity of Ref. [1]. The general AdS-wave solutions are derived and the different branches depending on their asymptotic behaviors are analyzed. In Sec.[V] we discuss some analogies between these configurations and their cousins arising in Topologically Massive Gravity; we also point out some differences. Besides, we consider the coupling between both massive models and derive the corresponding AdS-wave configurations. The nontrivial effects due to the inclusion of the topological mass term are analyzed in details in Sec. V.

\section{BRIEF INTRODUCTION TO ADS WAVES}

AdS waves are a special kind of exact gravitational waves propagating along AdS space. The first examples of exact gravitational waves in the presence of a cosmological constant were studied by García and Plebański [24]; see also Refs. [25, 26, 27]. Such solutions were based on generalizations of some algebraically special solutions previously found for the case of vanishing cosmological constant in Refs. 28, 29]. The algebraically special spacetimes are defined by the fact that their Weyl tensor has a multiple principal null direction. In addition, if this null direction is a Killing vector for the exact wave solutions then, in the case of a negative cosmological constant, one recovers the so-called Siklos spacetimes defining the AdS waves 30]. Siklos spacetimes allow an alternative characterization as a generalized Kerr-Schild transformation of AdS, which reinforces their interpretation as exact gravitational waves propagating on AdS space [31]. This means that their metrics can be written in terms of the AdS metric as follows

$$
g_{\mu \nu}=g_{\mu \nu}^{\mathrm{AdS}}-F k_{\mu} k_{\nu}
$$

where $k^{\mu}$ is a null geodesic field, and $F$ is an arbitrary function that is only constrained to be independent of the integral parameter along $k^{\mu}$; see Ref. [14] for a more detailed discussion.

Now, let us consider the three-dimensional case we are interested in. The $\mathrm{AdS}_{3}$ metric in Poincaré coordinates reads

$$
d s_{\mathrm{AdS}}^{2}=\frac{l^{2}}{y^{2}}\left(-2 d u d v+d y^{2}\right)
$$

where $l$ is the radius of AdS characterizing its constant scalar curvature $R=6 \Lambda=-6 / l^{2}$. Choosing as null geodesic field $k^{\mu} \partial_{\mu}=(y / l) \partial_{v}$, the Kerr-Schild transformation (11) allows to write the metric of the $\mathrm{AdS}_{3}$-waves as follows

$$
d s^{2}=\frac{l^{2}}{y^{2}}\left[-F(u, y) d u^{2}-2 d u d v+d y^{2}\right] .
$$

This metric is conformally related to that of a $p p$-wave. Nevertheless, it is worth pointing out that AdS-waves and $p p$-waves have different geometrical and physical properties. Let us be reminded of the fact that the term $p p$-wave stands for $p$ lane fronted gravitational waves with parallel rays. The fronts of the wave are defined by surfaces $u, v=$ const. in any number of dimension, and for the AdS-waves in higher dimensions they are not $p$ lanes but hyperboloids, having constant curvature proportional to $-1 / l^{2}$. Additionally, the null rays are defined by the field $\partial_{v}$, which is a Killing vector but not a closed 1-form, and thus the rays fail to be covariantly constant, namely parallel.

We will explore the existence of AdS waves configurations rigged by the New Massive Gravity of Ref. [1].

\section{THE ADS WAVES OF NEW MASSIVE GRAVITY}

The action of the New Massive Gravity is ${ }^{2}$

$$
S=\frac{1}{16 \pi G} \int d^{3} x \sqrt{-g}\left(R-2 \lambda-\frac{1}{m^{2}} K\right),
$$

where the quadratic contribution $K=R_{\mu \nu} R^{\mu \nu}-\frac{3}{8} R^{2}$ introduces the modification to standard gravity with cosmological constant $\lambda$, being $m$ the mass of the resulting massive degrees of freedom. The variation of (4) gives rise to the modified gravity equations of motion

$$
G_{\mu \nu}+\lambda g_{\mu \nu}-\frac{1}{2 m^{2}} K_{\mu \nu}=0
$$

where $G_{\mu \nu}=R_{\mu \nu}-\frac{1}{2} g_{\mu \nu}$ is the Einstein tensor and

$$
\begin{aligned}
K_{\mu \nu}= & 2 \square R_{\mu \nu}-\frac{1}{2} \nabla_{\mu} \nabla_{\nu} R-\frac{1}{2} \square R g_{\mu \nu}+4 R_{\mu \alpha \nu \beta} R^{\alpha \beta} \\
& -\frac{3}{2} R R_{\mu \nu}-K g_{\mu \nu},
\end{aligned}
$$

is a symmetric, conserved tensor that satisfies $g^{\mu \nu} K_{\mu \nu}=$ $K$. This condition on $K_{\mu \nu}$ implies that the trace of the equations of motion is a second order constraint, despite the fact these are equations of fourth order.

\footnotetext{
${ }^{2}$ We follow the conventions of [6] .
} 


\section{A. AdS-waves solutions}

For the New Massive Gravity to admit an $\mathrm{AdS}_{3}$ vacuum (2), a special constraint between the $\mathrm{AdS}_{3}$ radius $l$, the cosmological constant $\lambda$, and the mass parameter $m$ is needed. This fixes the value of the cosmological constant to be [1, [6]

$$
\lambda=-\frac{1}{l^{2}}\left(1+\frac{1}{4 l^{2} m^{2}}\right),
$$

which means the scale of the cosmological constant and the AdS radius only coincide in the General Relativity limit $m^{2} \rightarrow \pm \infty$.

The AdS-wave solutions (3) are meant to describe exact gravitational waves propagating along $\mathrm{AdS}_{3}$ spacetime of radius $l$, and thus we have to consider the same election (7) for the cosmological constant. With this choice for $\lambda$, the equations of motion (5) become a single differential equation for the wave profile $F$; namely

$$
\begin{aligned}
& {\left[y^{4} \partial_{y}^{4} F+2 y^{3} \partial_{y}^{3} F\right.} \\
& \left.\quad-\frac{\left(1+2 l^{2} m^{2}\right)}{2}\left(y^{2} \partial_{y}^{2} F-y \partial_{y} F\right)\right] \frac{\delta_{\mu}^{u} \delta_{\nu}^{u}}{2 l^{2} m^{2} y^{2}}=0,
\end{aligned}
$$

This is a fourth order Euler-Fuchs differential equation, which is easily solved by applying the standard substitution $F=y^{\alpha}$. The corresponding fourth-degree characteristic polynomial is

$$
\alpha(\alpha-2)\left((\alpha-1)^{2}-\frac{1+2 l^{2} m^{2}}{2}\right)=0 .
$$

Therefore, the generic solution for the wave profile is

$F(u, y)=F_{+}(u)\left(\frac{y}{l}\right)^{1+\sqrt{\frac{1+2 l^{2} m^{2}}{2}}}+F_{-}(u)\left(\frac{y}{l}\right)^{1-\sqrt{\frac{1+2 l^{2} m^{2}}{2}}}$,

where $F_{+}$and $F_{-}$are arbitrary integration functions that depend only on the retarded time $u$. Here and in what follows, we also use the fact that the homogeneous and quadratic dependence of the wave-front coordinate $y$ can be eliminated by coordinate transformations, see the detailed discussion in Ref. [14].

In addition to (10), we have to consider the possibility of having multiplicities in the roots of the characteristic polynomial (91). In this case, the power-law particular solutions fail to span the whole space of linearly independent solutions, and thus new additional logarithmic modes appear. Such multiplicities arise for the mass values $m^{2}= \pm 1 /\left(2 l^{2}\right)$. For $m^{2}=-1 /\left(2 l^{2}\right)$, there exists double multiplicity; the two roots exhibited in the generic solution (10) become one. Then, after discarding trivial behaviors, the wave profile at this point turns out to be given by

$$
F(u, y)=\frac{y}{l}\left[F_{1}(u) \ln \left(\frac{y}{l}\right)+F_{2}(u)\right] .
$$

On the other hand, for $m^{2}=+1 /\left(2 l^{2}\right)$ we find double multiplicity both for $\alpha=0$ and for $\alpha=2$, because in this case the roots of the generic solution (10) reduce to these values. Then, in this case we are left with the following solution

$$
F(u, y)=\ln \left(\frac{y}{l}\right)\left[F_{1}(u)\left(\frac{y}{l}\right)^{2}+F_{2}(u)\right] .
$$

Finally, for $m^{2}<-1 /\left(2 l^{2}\right)$ the relevant roots of (9) take complex values, and the solution becomes

$$
\begin{aligned}
F(u, y)= & \frac{y}{l}\left\{F_{1}(u) \sin \left[l \sqrt{-\frac{1}{2 l^{2}}-m^{2}} \ln \left(\frac{y}{l}\right)\right]\right. \\
& \left.+F_{2}(u) \cos \left[l \sqrt{-\frac{1}{2 l^{2}}-m^{2}} \ln \left(\frac{y}{l}\right)\right]\right\} .
\end{aligned}
$$

The configurations given by (10)-(13) represent the $\mathrm{AdS}_{3}$-wave solutions to the New theory of Massive Gravity [1]. In Section IV we will discuss the analogy between these solutions and those arising in the context of the Topologically Massive Gravity. But, first, let us comment on the asymptotic behavior of the solutions we just described.

\section{B. The asymptotically $\mathrm{AdS}_{3}$ sector}

As mentioned, AdS-waves are Siklos spacetimes 30] that can be thought of as gravitational wave profiles propagating on AdS spacetime [31]. Here, we will show that, in addition, some of these wave solutions of New Massive Gravity are also asymptotically $\mathrm{AdS}_{3}$.

New Massive Gravity in $\mathrm{AdS}_{3}$ has been recently studied in [1, [1, [6] . According to $\mathrm{AdS}_{3} / \mathrm{CFT}_{2}$ correspondence, the theory formulated in $\mathrm{AdS}_{3}$ would be dual to a twodimensional conformal field theory with central charge given by

$$
c=\frac{3 l}{2 G}\left(1-\frac{1}{2 m^{2} l^{2}}\right) .
$$

This value for the central charge is easily obtained by standard means 32]. From this we observe that something special happens at $m^{2}=1 /\left(2 l^{2}\right)$, where $c$ vanishes. Likely, the unitarity of the theory (when sufficiently relaxed boundary conditions are considered) would demand the bound $m^{2}>1 /\left(2 l^{2}\right)$. Let us discuss the different asymptotic behaviors in relation to this bound.

First, let us take a look at solutions (10). The wave solution turns out to be an asymptotically $\mathrm{AdS}_{3}$ spacetime if $F_{-}=0$ and $m^{2}>1 /\left(2 l^{2}\right)$. That is, the solution is asymptotically $\mathrm{AdS}_{3}$ according to Brown-Henneaux boundary conditions [10], which, in these coordinates, are defined by the next-to-leading behavior

$$
g_{\mu \nu}=g_{\mu \nu}^{\mathrm{AdS}}+h_{\mu \nu}
$$


where $g_{\mu \nu}^{\mathrm{AdS}}$ is given by (2), while the components of the perturbation $h_{\mu \nu}$ are of order $h_{u u} \sim h_{u v} \sim h_{v v} \sim h_{y y} \sim$ $\mathcal{O}(1)$, and $h_{u y} \sim h_{v y} \sim \mathcal{O}(y)$.

On the other hand, at the critical value $m^{2}=1 /\left(2 l^{2}\right)$, solution (12) turns out to be compatible with the weakened (logarithmic) $\mathrm{AdS}_{3}$ asymptotic behavior discussed in Refs. [11, 12, 22, 23], which amount to relax boundary conditions as $h_{u u} \sim \mathcal{O}(\ln y)$ and $h_{u y} \sim \mathcal{O}(y \ln y)$. These weakened $\mathrm{AdS}_{3}$ boundary conditions were originally discussed within the context of Topologically Massive Gravity, where the analog of solution (12) given in Refs. [13, 14] is Eq. (45) below, and it was recently argued that they might play an important role in New Massive Gravity too [6]. It has been known for a while that, for certain particular points of the space of parameters of a given theory, the concept of asymptotically $\mathrm{AdS}_{3}$ space may be consistently extended to incorporate a larger class of geometries [33]. This issue has played an important role in recent discussions on Topologically Massive Gravity [23].

\section{ANALOGIES WITH TOPOLOGICALLY MASSIVE GRAVITY}

It is interesting to notice that all the branches of solutions discussed above, except the complex one (13), have their counterparts in Topologically Massive Gravity [13, 14, 15, 16, 17, 18]. In particular, the critical cases $m^{2}= \pm 1 /\left(2 l^{2}\right)$ deserve a particular attention because these are reminiscent of the chiral values $\mu= \pm 1 / l$ of Topologically Massive Gravity, with $\mu$ being the topological mass. These points of the space of parameters were shown to be special in what regards to the massive behavior of $\mathrm{AdS}_{3}$-wave solutions [14]. More recently, the points $\mu= \pm 1 / l$ appeared to be relevant also for the discussion about the chiral gravity conjecture [19]; see also [11, 17, 20, 23] and references therein.

The purpose of this section is to discuss this and other analogies between the AdS-wave solutions of New Massive Gravity and those of Topologically Massive Gravity.

\section{A. The Schrödinger invariant sector}

Recently, a generalization of the AdS/CFT correspondence has been proposed in the context of non-relativistic conformal field theories. The basic idea is that geometries whose isometry group agrees with the non-relativistic conformal group, namely the Schrödinger group, could represent gravity duals for systems of condensed matter physics [34, 35]. The Schrödinger group is defined as the maximal group of symmetries which leave invariant the Schrödinger equation for a free particle [36, 37], and can be thought of as the semi direct product of $\mathrm{SL}(2, \mathbb{R})$ with the connected static Galilei group. The set of Schrödinger transformations are given by the standard
Galilei transformations augmented by the time dilatation and a special conformal transformation.

Because of the holographic applications to nonrelativistic CFTs, the search of theories that admit as solutions Schrödinger-invariant backgrounds has attracted much attention recently. Moreover, it is worth mentioning that the AdS-waves of Topologically Massive Gravity 14] contains Schrödinger invariant solutions at the special point $\mu=3 / l[38]$. These solutions correspond to the null warped- $\mathrm{AdS}_{3}$ spacetimes of [21]. Here, let us show that our generic solution (10) of New Massive Gravity may exhibit the Schrödinger isometry too. In fact, if one takes one of the arbitrary functions $F_{ \pm}$to be a constant and the other one to be zero, then the solution (10) simply reads

$$
F(y)=F_{0}\left(\frac{y}{l}\right)^{-2 \nu}
$$

where $F_{0}$ is an arbitrary constant and $\nu=-\alpha / 2$ is called the "dynamical exponent" in this context. Interesting enough, the isometry group of metric (3) for solution (16) gets enhanced, exhibiting the so-called partial Schrödinger group, which is the group of all Schrödinger transformations except the special conformal transformation. The partial Schrödinger symmetry is realized by the Killing vectors

$$
\begin{aligned}
H & =\partial_{v}, \quad N=\partial_{u}, \\
D & =(1+\nu) v \partial_{v}+(1-\nu) u \partial_{u}+y \partial_{y} .
\end{aligned}
$$

In addition, the particular election $F_{+}=0$ and $F_{-}=$ const. allows the special case $\nu=1$, which corresponds to

$$
m^{2}=\frac{17}{2 l^{2}}
$$

and the solution exhibits the full Schrödinger symmetry, i.e. the isometry group is augmented by the Killing vector

$$
C=v^{2} \partial_{v}+\frac{1}{2} y^{2} \partial_{u}+y v \partial_{y} .
$$

This critical point (17) is analogous to the point $\mu=$ $3 / l$ of the Topologically Massive Gravity (see Eq. (32) below). This reinforces the resemblance between both theories.

\section{B. AdS waves as massive scalar modes: log waves saturate the BF bound}

Another interesting property of solutions (10) is that the profile function $F$ behaves exactly as a massive scalar mode, as it satisfies the Klein-Gordon equation

$$
\square F=m_{\text {eff }}^{2} F,
$$

with effective mass given by

$$
m_{\mathrm{eff}}^{2}=m^{2}-\frac{1}{2 l^{2}} .
$$


This shifting of the bare mass $m$ by a term proportional to the curvature of the $\mathrm{AdS}_{3}$ space is also observed in the case of $\mathrm{AdS}_{3}$-waves of Topologically Massive Gravity, where the effective mass is found to be

$$
\mu_{\mathrm{eff}}^{2}=\mu^{2}-\frac{1}{l^{2}},
$$

(see Ref. [14] for details). However, the New Massive Gravity profile (10) describes in fact the superposition of two scalar modes, in contrast with Topologically Massive Gravity, for which a single mode arises (see Eq. (32) later). Actually, at this level it may seem artificial to make a distinction between the "two modes" appearing in Eq. (10) since, after all, they have the same effective mass (19). However, we will see in the next section that the inclusion of a topologically massive term (i.e. the Chern-Simons gravitational term) breaks this mass degeneracy and thus the distinction between the two modes ultimately makes sense.

In the case of the New Massive Gravity, and for the special case $m^{2}=-1 /\left(2 l^{2}\right)$, the profile (11) also describes the superposition of two exact massive scalar modes, each one satisfying

$$
\square F=-\frac{1}{l^{2}} F .
$$

This case does not differ from the generic one (18) since the effective mass becomes $m_{\text {eff }}^{2}=-1 / l^{2}$ and thus corresponds to the mass given by (19). Interesting enough, we find that this value for the effective mass exactly saturates the Breitenlohner-Freedman bound for the mass of a scalar field in the $\mathrm{AdS}_{3}$ space where the wave is propagating on [39, 40].

The result (20) for the case $m^{2}=-1 /\left(2 l^{2}\right)$ is in contrast with what happens in Topologically Massive Gravity, where none of the logarithmic solutions that appear at $\mu= \pm 1 / l$ (see Eqs. (42) and (45) below) satisfy the Klein-Gordon equation. The analogy with Topologically Massive Gravity is thus manifested at the other critical point, $m^{2}=+1 /\left(2 l^{2}\right)$. At this point, the wave profile (12) does not satisfy a Klein-Gordon equation, and, as mentioned before, this is precisely the point of the space of parameters where the asymptotically $\mathrm{AdS}_{3}$ spaces admit logarithmic branches [6] similar to those appearing for Topologically Massive Gravity at the chiral point $\mu=-1 / l,\left[11,[12]^{3}\right.$. Also, it is interesting to notice that the New Massive Gravity profile (12) is exactly a superposition of the critical profiles (42) and (45) of Topologically Massive Gravity

$$
F=\left.F_{\mathrm{TMG}}\right|_{\mu=-1 / l}+\left.F_{\mathrm{TMG}}\right|_{\mu=+1 / l} .
$$

We remark that, despite the fact the profile function $F$ in Topologically Massive Gravity at the points $\mu= \pm 1 / l$

\footnotetext{
${ }^{3}$ Our definition of the topological mass is minus the one of those references, where the chiral point occurs for $\mu=+1 / l$.
}

do not obey the Klein-Gordon equation, it can be used to generate the following scalar modes,

$$
\left.\frac{l}{y} F_{\mathrm{TMG}}\right|_{\mu=-1 / l}, \quad \text { and }\left.\quad \frac{y}{l} F_{\mathrm{TMG}}\right|_{\mu=+1 / l},
$$

which do satisfy the Klein-Gordon equation (20) saturating the Breitenlohner-Freedman bound. That is, one can interpret the profile (12) of New Massive Gravity at the critical point $m^{2}=+1 /\left(2 l^{2}\right)$ as a local superposition

$$
F=\frac{y}{l}\left[\left.\frac{l}{y} F\right|_{F_{2}=0}\right]+\frac{l}{y}\left[\left.\frac{y}{l} F\right|_{F_{1}=0}\right],
$$

of exact massive scalar modes (those between brackets) which saturate the Breitenlohner-Freedman bound.

So far, we have discussed the analogies between New Massive Gravity and Topologically Massive Gravity. Now, let us move to analyze what happens when these two theories are brought together.

\section{TURNING ON A TOPOLOGICAL CONTRIBUTION}

In this section, we analyze the effect of turning on the Chern-Simons topological term in the gravitational action (4), and see how it affects the existence and properties of AdS-wave configurations we discussed so far. The inclusion of the topologically massive term in the action amounts to add the Cotton tensor ${ }^{4}$ [2, 3]

$$
C^{\mu \nu}=\eta^{\mu \alpha \beta} \nabla_{\alpha}\left(R_{\beta}^{\nu}-\frac{1}{4} R \delta_{\beta}^{\nu}\right),
$$

to the equations of motion (5). The resulting field equations read [1]

$$
G_{\mu \nu}+\lambda g_{\mu \nu}-\frac{1}{2 m^{2}} K_{\mu \nu}+\frac{1}{\mu} C_{\mu \nu}=0,
$$

where the coupling constant $\mu$ stands for the topological mass. It is known that the Cotton tensor vanishes for constant curvature configurations. Therefore, in order for the $\mathrm{AdS}_{3}$ metric (2) to be a solution of the generalized equations (25), the constraint between the cosmological constant $\lambda$, the AdS radius $l$, and the mass $m$, must be exactly the same as in Eq. (7).

For an AdS-wave (3), the only nonvanishing component of the Cotton tensor is $C_{u u}$, and it is proportional to the third derivative of the wave profile with respect to the wave-front coordinate $y$. The resulting single equation is again of the Euler-Fuchs type; namely

$$
\begin{aligned}
& {\left[y^{4} \partial_{y}^{4} F+\left(2-\frac{l m^{2}}{\mu}\right) y^{3} \partial_{y}^{3} F\right.} \\
& \left.\quad-\frac{\left(1+2 l^{2} m^{2}\right)}{2}\left(y^{2} \partial_{y}^{2} F-y \partial_{y} F\right)\right] \frac{\delta_{\mu}^{u} \delta_{\nu}^{u}}{2 l^{2} m^{2} y^{2}}=0 .
\end{aligned}
$$

\footnotetext{
4 Here, $\eta_{\mu \alpha \beta}$ corresponds to the volume 3-form, with $\eta_{u v y}=\sqrt{-g}$ $\left(\eta^{u v y}=-1 / \sqrt{-g}\right)$.
} 
The characteristic polynomial is now given by

$$
\alpha(\alpha-2)\left[\left(\alpha-1-\frac{l m^{2}}{2 \mu}\right)^{2}-\frac{1+2 l^{2} m^{2}}{2}-\frac{l^{2} m^{4}}{4 \mu^{2}}\right]=0 .
$$

Below, we will analyze all the possible solutions.

\section{A. Topological mass splitting}

According to (27), the generic solution is given by

$$
\begin{aligned}
F(u, y)= & F_{+}(u)\left(\frac{y}{l}\right)^{1+\frac{l m^{2}}{2 \mu}+\sqrt{\frac{1+2 l^{2} m^{2}}{2}+\frac{l^{2} m^{4}}{4 \mu^{2}}}} \\
& +F_{-}(u)\left(\frac{y}{l}\right)^{1+\frac{l m^{2}}{2 \mu}-\sqrt{\frac{1+2 l^{2} m^{2}}{2}+\frac{l^{2} m^{4}}{4 \mu^{2}}}} .
\end{aligned}
$$

This generalizes the generic profile of New Massive gravity (10). It represents the superposition of two exact scalar modes, both satisfying a Klein-Gordon equation (18). These modes are given by $F_{-}=0\left(\right.$ resp. $\left.F_{+}=0\right)$ with effective masses given respectively by

$$
m_{\mathrm{eff} \pm}^{2}=\left(\frac{m^{2}}{2 \mu} \pm \sqrt{\frac{m^{4}}{4 \mu^{2}}+m^{2}+\frac{1}{2 l^{2}}}\right)^{2}-\frac{1}{l^{2}} .
$$

From this we observe that the physical effect of including the topological term is that of breaking the degeneracy in the mass spectrum (19) for the two scalar modes of pure New Massive Gravity (10). In other words, the topological term produces the following mass splitting between the two generic gravitational states

$$
\Delta m_{\mathrm{eff}}^{2}=\frac{2 m^{2}}{\mu} \sqrt{\frac{m^{4}}{4 \mu^{2}}+m^{2}+\frac{1}{2 l^{2}}} .
$$

It is interesting that one has access to this fine structure effect beyond the perturbative level.

The spacetime configurations (28) also contain sectors enjoying the partial Schrödinger isometry as in Eq. (16), while the full Schrödinger symmetry is exhibited this time for

$$
m^{2}=\frac{17 \mu}{2 l^{2}(\mu-3 / l)} .
$$

Notice that the generic formulas of Sec. III are obtained from the previous ones in the limit $\mu \rightarrow \pm \infty$. It is also remarkable that the critical value for which the AdS-waves solutions of Topologically Massive Gravity are Schrödinger invariant, i.e. $\mu=3 / l$, is also recovered from the mass (31) in the limit $m^{2} \rightarrow \pm \infty$. Actually, in this limit, one of the two modes in Eq. (28) diverges/vanishes and has no analogue in Topologically Massive Gravity, while the other mode corresponds precisely to the single generic mode that appears in Topologically Massive Gravity [13, 14]; namely

$$
F_{\mathrm{TMG}}(u, y)=F_{1}(u)\left(\frac{y}{l}\right)^{1-l \mu} .
$$

This two-to-one correspondence between the modes of both theories is a common feature of this transition and is easily understood by taking into account that, unlike in New Massive Gravity, parity is broken in Topologically Massive Gravity, and this fact forces the latter theory to select only one of the two modes arising in the former.

\section{B. Logarithmic Branches}

Now, let us discuss the cases allowing multiplicities in the roots of the characteristic polynomial (27). These cases are those where logarithmic branches arise.

The roots corresponding to the generic solution (28) reduce to a single one for the two following families of mass values

$$
m^{2}=2 \mu^{2}\left(-1 \pm \sqrt{1-\frac{1}{2 l^{2} \mu^{2}}}\right) .
$$

This double multiplicity leads to two new families of solutions; namely

$$
\begin{aligned}
F(u, y)= & \left(\frac{y}{l}\right)^{1+l \mu\left[-1 \pm \sqrt{1-1 /\left(2 l^{2} \mu^{2}\right)}\right]} \\
& \times\left[F_{1}(u) \ln \left(\frac{y}{l}\right)+F_{2}(u)\right] .
\end{aligned}
$$

For the upper sign, and taking the limit $\mu \rightarrow \pm \infty$, the mass goes like

$$
m^{2}=-\frac{1}{2 l^{2}}+\mathcal{O}\left(\frac{1}{\mu^{2}}\right)
$$

and then one recovers the first critical solution (11), studied in Sec. III] The same limit is divergent for the lowersign since

$$
m^{2}=-4 \mu^{2}+\frac{1}{2 l^{2}}+\mathcal{O}\left(\frac{1}{\mu^{2}}\right),
$$

Actually, this describes the transition to the standard $\mathrm{AdS}_{3}$ Einstein gravity instead of to pure New Massive Gravity. It is known that AdS waves are locally trivial in this context [14], and consequently, one mode vanishes and the other diverges in the above limit.

For the lower-sign family, and for the topological mass taking the value $\mu=3 /(4 l)$ (resp. $\mu=-3 /(4 l)$ ), the root $\alpha=0$ (resp. $\alpha=2$ ) becomes triple and then the solutions in these cases respectively read

$$
F(u, y)=\ln \left(\frac{y}{l}\right)\left[F_{1}(u) \ln \left(\frac{y}{l}\right)+F_{2}(u)\right],
$$

and

$$
F(u, y)=\left(\frac{y}{l}\right)^{2} \ln \left(\frac{y}{l}\right)\left[F_{1}(u) \ln \left(\frac{y}{l}\right)+F_{2}(u)\right] .
$$

Another critical value for the mass is given by

$$
m^{2}=\frac{\mu}{2 l^{2}(\mu-1 / l)},
$$


where the value $\alpha=0$ turns out to be a double root. The solution, for a generic value of the topological mass $\mu \neq 3 /(4 l),{ }^{5}$ is

$$
F(u, y)=F_{1}(u)\left(\frac{y}{l}\right)^{(4 l \mu-3) /[2(l \mu-1)]}+F_{2}(u) \ln \left(\frac{y}{l}\right) .
$$

In the limit $\mu \rightarrow \pm \infty$, the exponent in the first term above takes the value 2 and the associated mode can be eliminated by coordinate transformations. Besides, since the associated mass (39) behaves like

$$
m^{2}=\frac{1}{2 l^{2}}+\mathcal{O}\left(\frac{1}{\mu}\right),
$$

one recovers just one of the two modes of the critical solution (12). Moreover, from the mass expression (39), we notice that the Topologically Massive Gravity limit, $m^{2} \rightarrow \pm \infty$, is achieved for $\mu \rightarrow 1 / l$. In this limit, the mode associated to the power law in (40) diverges/vanishes and has no analogue in Topologically Massive Gravity. In contrast, the logarithmic mode survives and then one recovers the single critical mode of Topologically Massive Gravity with $\mu=1 / l[13,14]$

$$
\left.F_{\mathrm{TMG}}(u, y)\right|_{\mu=+1 / l}=F_{2}(u) \ln \left(\frac{y}{l}\right) .
$$

The last example for which double multiplicity arises is the point

$$
m^{2}=\frac{\mu}{2 l^{2}(\mu+1 / l)}
$$

where the root $\alpha=2$ becomes double. The corresponding solution for a generic value of the topological mass, as long as $\mu \neq-3 /(4 l)$, is expressed by

$$
F(u, y)=F_{1}(u)\left(\frac{y}{l}\right)^{2} \ln \left(\frac{y}{l}\right)+F_{2}(u)\left(\frac{y}{l}\right)^{1 /[2(l \mu+1)]} .
$$

In the limit $\mu \rightarrow \pm \infty$, the power-law contribution in the second term of (44) can be eliminated by coordinate transformations and the associated mass (43) behaves as in Eq. (41). This limiting case allows to recover the remaining mode of the critical solution (12) which was absent in the previous solution. Taking a look at the Topologically Massive Gravity limit $\left(m^{2} \rightarrow \pm \infty\right)$ in (43) one notices that it is in correspondence with the limit $\mu \rightarrow-1 / l$. Here again, the mode associated to the powerlaw dependence in Eq. (44) diverges/vanishes and has no analogue in Topologically Massive Gravity. The logarithmic mode remains untouched and it becomes exactly the single critical mode allowed for $\mu=-1 / l[13,14]$; namely

$$
\left.F_{\mathrm{TMG}}(u, y)\right|_{\mu=-1 / l}=F_{1}(u)\left(\frac{y}{l}\right)^{2} \ln \left(\frac{y}{l}\right) \text {. }
$$

\footnotetext{
${ }^{5}$ In what follows we exclude the cases $\mu= \pm 3 /(4 l)$ since they have triple multiplicity and were already considered, see Eqs. (37) and (38).
}

These cases exhaust all the AdS-wave solutions (3) one finds for the theory (25).

\section{CONCLUSIONS}

In this paper, we have studied AdS-wave configurations in three-dimensional massive gravities.

The first model we considered was the New Massive Gravity recently proposed by Bergshoeff, Hohm, and Townsend, in Ref. [1]. In addition to the cosmological constant, this model has a mass parameter $m$, which is the coupling constant of the higher-curvature terms that supplement the Einstein-Hilbert action. For this theory, we considered AdS-wave configurations, which correspond to exact solutions that can be thought of as waves propagating on $\mathrm{AdS}_{3}$ spacetime of radius $l$. These AdSwave solutions are characterized by a function $F(u, y)$, which describes the profile of the wave, and depends on the retarded time $u$ and on the front-wave coordinate $y$.

We have exhaustively explored the space of solutions of this kind and, in particular, we have shown that special features occur at the critical values $m^{2}= \pm 1 /\left(2 l^{2}\right)$. At these points, solutions with logarithmic fall-off in the Poincaré radial coordinate $y$ arise. This resembles what happens in the case of Topologically Massive Gravity at the chiral point $\mu= \pm 1 / l\left[14\right.$. At $m^{2}=+1 /\left(2 l^{2}\right)$, one finds that asymptotically $\mathrm{AdS}_{3}$ exact solutions obeying the weakened fall-off proposed in [11, 12] for Topologically Massive Gravity appear; a fact that is suggested by the linearized analysis performed in Ref. [6] for New Massive Gravity. This special mass coincides with the point of the space of parameters at which the central charge of the dual $\mathrm{CFT}_{2}$ vanishes. This naturally leads one to the conjecture that, likely, the New Massive Gravity of [1] at the point $m^{2}=1 /\left(2 l^{2}\right)$ is dual to a two-dimensional logarithmic conformal field theory if sufficiently weakened $\mathrm{AdS}_{3}$ asymptotic conditions are considered. For the range of parameters $m^{2}>1 /\left(2 l^{2}\right)$, however, asymptotically $\mathrm{AdS}_{3}$ solutions obeying stronger Brown-Henneaux boundary conditions arise, and the theory is likely unitary.

We also found solutions whose isometry corresponds to the Schrödinger symmetry group. These geometries exist for $m^{2}>1 /\left(2 l^{2}\right)$, and are analogous to those that were recently considered in the context of the nonrelativistic version of the AdS/CFT duality [34, 35]. In particular, the full Schrödinger symmetry is achieved for $m^{2}=17 /\left(2 l^{2}\right)$.

For all the values $m^{2} \neq 1 /\left(2 l^{2}\right)$ the profile function $F$ behaves as a massive scalar excitation, as it satisfies the Klein-Gordon equation with effective mass $m_{\text {eff }}^{2}=m^{2}-$ $1 /\left(2 l^{2}\right)$. In fact, the profiles describe two exact scalar modes sharing the same mass. In particular, for the case $m^{2}=-1 /\left(2 l^{2}\right)$ this modes saturates the BreitenlohnerFreedman bound for a massive particle on the $\mathrm{AdS}_{3}$ space where the wave is propagating on.

We also considered the New Massive theory of Grav- 
ity coupled to Topologically Massive Gravity. This introduces a second mass scale $\mu$ in the theory. For this model, we have exhaustively explored the space of AdS-wave solutions, and we have shown that different branches with different asymptotic behaviors arise. We discussed the effects of turning on the gravitational Chern-Simons term: This induces a mass splitting that breaks the mass degeneracy present in the generic modes of the New Massive Gravity waves. Additionally, several generalization of the previous results are obtained, for example, full Schrödinger invariant backgrounds are obtained now for $m^{2}=17 \mu /\left[2 l^{2}(\mu-3 / l)\right]$. We also analyze the different limits both to New Massive Gravity and to Topologically Massive Gravity obtaining consistent results. The interplay between the parity preserving and the parity violating Lagrangians is also discussed.
A recent paper [41] studies the linearized solutions of New Massive Gravity coupled to Topologically Massive Gravity in $\mathrm{AdS}_{3}$. The asymptotic behaviors of the exact solutions we have found here realize some of the linearized solutions of Ref. [41].

\section{Acknowledgments}

The authors are grateful to A. Garbarz, A. García and J. Oliva for useful discussions. This work has been partially supported by grant 1090368 from FONDECYT, by grant UBACyT X861 from UBA, and by grants 82443 and 45946-F from CONACyT.
[1] E. A. Bergshoeff, O. Hohm and P. K. Townsend, "Massive Gravity in Three Dimensions," arXiv:0901.1766 [hep-th].

[2] S. Deser, R. Jackiw and S. Templeton, Annals Phys. 140, 372 (1982) [Erratum-ibid. 185, 406.1988 APNYA,281,409 (1988 APNYA,281,409-449.2000)].

[3] S. Deser, R. Jackiw and S. Templeton, Phys. Rev. Lett. 48, 975 (1982).

[4] G. Clement, "Warped $A d S_{3}$ black holes in new massive gravity," arXiv:0902.4634 [hep-th].

[5] Y. Liu and Y. Sun, "Note on New Massive Gravity in $A d S_{3}, "$ arXiv:0903.0536 [hep-th].

[6] Y. Liu and Y. W. Sun, "Consistent Boundary Conditions for New Massive Gravity in $A d S_{3}$," arXiv:0903.2933 [hepth].

[7] M. Nakasone and I. Oda, "On Unitarity of Massive Gravity in Three Dimensions," arXiv:0902.3531 [hep-th].

[8] M. Nakasone and I. Oda, "Massive Gravity with Mass Term in Three Dimensions," arXiv:0903.1459 [hep-th].

[9] J. Oliva, D. Tempo and R. Troncoso, in preparation.

[10] J. D. Brown and M. Henneaux, Commun. Math. Phys. 104, 207 (1986).

[11] D. Grumiller and N. Johansson, JHEP0807:134,2008, arXiv:0805.2610 [hep-th]; Int. J. Mod. Phys. D17 2367 (2009); arXiv:0808.2575 [hep-th].

[12] M. Henneaux, C. Martinez and R. Troncoso, "Asymptotically anti-de Sitter spacetimes in topologically massive gravity," arXiv:0901.2874 [hep-th].

[13] E. Ayón-Beato and M. Hassaine, Annals Phys. 317, 175 (2005), arXiv:hep-th/0409150

[14] E. Ayón-Beato and M. Hassaine, Phys. Rev. D 73, 104001 (2006), arXiv:hep-th/0512074.

[15] T. Dereli and O. Sarioglu, "Topologically massive gravity and black holes in three dimensions," arXiv:gr-qc/0009082

[16] S. Olmez, O. Sarioglu and B. Tekin, Class. Quant. Grav. 22, 4355 (2005), arXiv:gr-qc/0507003.

[17] S. Carlip, S. Deser, A. Waldron and D. K. Wise, Phys. Lett. B 666, 272 (2008), arXiv:0807.0486 [hep-th].

[18] G. W. Gibbons, C. N. Pope and E. Sezgin, Class. Quant. Grav. 25, 205005 (2008), arXiv:0807.2613 [hep-th].

[19] W. Li, W. Song and A. Strominger, JHEP 0804, 082
(2008), arXiv:0801.4566 [hep-th].

[20] S. Carlip, S. Deser, A. Waldron and D. K. Wise, Class. Quant. Grav. 26 (2009) 075008, arXiv:0803.3998 [hep-th]. A. Strominger, "Simple Proof of the Chiral Gravity Conjecture", arXiv:0808.0506 [hep-th]. S. Carlip, JHEP 0810, 078 (2008), arXiv:0807.4152 [hepth]. G. Giribet, M. Kleban and M. Porrati, JHEP 10 (2008) 045, arXiv arXiv:0807.4703 [hep-th]. S. Carlip, S. Deser, A. Waldron and D. K. Wise, Mu-in Park, JHEP 09 (2008) 084, arXiv:0805.4328 [hep-th]. M. Blagojević and B. Cvetković, "Canonical structure of topologically massive gravity with a cosmological constant," arXiv:0812.4742 [hep-th]. D. Grumiller, R. Jackiw and N. Johansson, "Canonical analysis of cosmological topologically massive gravity at the chiral point", in Wolfgang Kummer Memorial Volume, arXiv:0806.4185 [hep-th]. S. Carlip, JHEP 10 (2008) 078, arXiv:0807.4152 [hep-th].

[21] D. Anninos, W. Li, M. Padi, W. Song and A. Strominger, "Warped $\mathrm{AdS}_{3}$ Black Holes", arXiv:0807.3040 [hep-th].

[22] A. Garbarz, G. Giribet and Y. Vásquez, Phys. Rev. D 79 (2009) 044036, arXiv:0811.4464 [hep-th].

[23] A. Maloney, W. Song and A. Strominger, "Chiral Gravity, Log Gravity and Extremal CFT," arXiv:0903.4573 [hep-th].

[24] A. García and J. Plebański, J. Math. Phys. 22, 2655 (1981).

[25] H. Salazar, A. García, and J. Plebański, J. Math. Phys. 24, 2191 (1983).

[26] A. García, Nuovo Cim. B 78, 255 (1983).

[27] I. Ozsvath, I. Robinson, and K. Rozga, J. Math. Phys. 26, 1755 (1985).

[28] W. Kundt, Z. Phys. 163, 77 (1961).

[29] I. Robinson and A. Trautman, Phys. Rev. Lett. 4, 431 (1960).

[30] S.T.C. Siklos, in: Galaxies, axisymmetric systems and relativity, ed. M.A.H. MacCallum (Cambridge Univ. Press, Cambridge 1985).

[31] J. Podolsky, Class. Quant. Grav. 15, 719 (1998), arXiv:gr-qc/9801052

[32] P. Kraus, Lect. Notes Phys. 755 (2008) 193, arXiv:hep-th/0609074

[33] M. Henneaux, C. Martínez, R. Troncoso and J. Zanelli, 
Phys. Rev. D 70044034 (2004), arXiv:hep-th/0404236. Annals Phys. 322 (2007) 824, arXiv:hep-th/0603185

[34] D. T. Son, Phys. Rev. D 78, 046003 (2008), arXiv:0804.3972 [hep-th].

[35] K. Balasubramanian and J. McGreevy, Phys. Rev. Lett. 101, 061601 (2008), arXiv:0804.4053 [hep-th].

[36] R. Jackiw, Phys. Today, 25, 23 (1972).

[37] U. Niederer, Helv. Phys. Acta 45, 802 (1972). C. R. Hagen, Phys. Rev. D5, 377 (1972).
[38] C. Duval, M. Hassaine and P. A. Horvathy, Annals Phys. 324, 1158 (2009), arXiv:0809.3128 [hep-th].

[39] P. Breitenlohner and D. Z. Freedman, Phys. Lett. B 115, 197 (1982); Annals Phys. 144, 249 (1982).

[40] L. Mezincescu and P. K. Townsend, Annals Phys. 160, 406 (1985).

[41] Y. Liu and Y.W. Sun, "On the generalized massive gravity in $\mathrm{AdS}_{3}$ ", arXiv:0904.0403 [hep-th]. 